\title{
Formation Processes of Metal-Rich Tribofilm on the Counterface During Sliding Against Metal/Diamondlike-Carbon Nanocomposite Coatings
}

\author{
Minoru Goto $^{1)^{*}}$, Kosuke Ito ${ }^{2)}$, Julien Fontaine ${ }^{3)}$, Takanori Takeno ${ }^{4)}$, Hiroyuki Miki ${ }^{5)}$ and Toshiyuki Takagi ${ }^{6)}$ \\ ${ }^{1)}$ Department of Mechanical Engineering, Ube National College of Technology \\ 2-14-1 Tokiwadai, Ube 755-8555, Japan \\ ${ }^{2)}$ College of Engineering, Nihon University \\ 1 Nakagawara, Tokusada, Tamuramachi, Koriyama 963-8642, Japan \\ ${ }^{3}$ Laboratoire de Tribologie et Dynamique des Systèmes, Ecole Centrale de Lyon \\ 36 Avenue Guy de Collongue, Ecully Cedex 69134, France \\ ${ }^{4)}$ Graduate School of Engineering, Tohoku University \\ 6-6-01 Aramaki Aza, Aoba-ku, Sendai 980-8579, Japan \\ ${ }^{5)}$ Frontier research Institute for Interdisciplinary Science, Tohoku University \\ 6-3 Aramaki Aza, Aoba-ku, Sendai 980-8577, Japan \\ ${ }^{6)}$ Institute of Fluid Science, Tohoku University \\ 2-1-1 Katahira, Aoba-ku, Sendai 980-8577, Japan \\ *Corresponding author: mi-goto@ube-k.ac.jp
}

( Manuscript received 16 June 2015; accepted 4 August 2015; published 31 October 2015 )

\begin{abstract}
Formation processes of metal-rich tribofilm on the counterface during sliding against metal/diamondlike-carbon nanocomposite coatings have been investigate. The hybridization between DLC matrix and $\mathrm{Cu}$ or $\mathrm{Ag}$ provides good electric conductivity to solid lubricant films. Metal-rich tribofilms are formed on the counterface of both Cu-DLC and Ag-DLC during sliding, which is important factor to obtain excellent tribological performance as well as good electric conductivity. Such tribofilms are almost pure $\mathrm{Cu}$ and Ag. The origin of the tribofilm is the metal clusters in the Me-DLC, and the adhered $\mathrm{Cu}$ and Ag clusters on the counterfaces are possibly sintered and refined during iterative plastic deformation in the frictional interface which generates high pressure and high shear rate.
\end{abstract}

Keywords: $\mathrm{Cu}-\mathrm{DLC}, \mathrm{Ag}-\mathrm{DLC}$, nanocomposite, tribology, metal-rich tribofilm, sintering, electric contact resistance

\section{Introduction}

Diamond-like carbon (DLC) coatings have been motivating a lot of researches because of their excellent tribological characteristics [1]. The DLC, however, has some disadvantages such as a lack of electrical conductivity and strong sensitivity of tribological behavior to the sliding conditions. The robustness of tribological properties against the operating conditions of the tribo-system would be desirable, not only for many kinds of solid lubricant, but also for DLC coatings. In addition to that, if the DLC can obtain electrical conductivity, it would be applicable to electrical sliding contacts, which need both low friction loss and high durability.

Adding metals to DLC coatings is thus considered as a powerful method to improve electrical conductivity as well as tribological properties. Copper and silver are two of the potential candidates as addition elements, because of their low electrical resistivity. In addition, Ag film is known as a practical solid lubricant for aerospace applications [2]. In recent years, we have reported on the tribological properties of metal/DLC nanocomposite coatings (Me-DLC) [3-8]. Especially, copper/DLC nanocomposite (Cu-DLC) and silver/DLC nanocomposite (Ag-DLC) coatings exhibited good electrical conductivity. The $\mathrm{Cu}$-DLC showed stable friction coefficient under different loading conditions, whereas the friction coefficient of Ag-DLC varied depending on the applied normal load and the counter material. In both cases, a selective metal-rich transfer layer was formed on the counterface when the stable friction coefficient and sufficient durability of the films were obtained. The film generated in the frictional interface by tribological interactions is generally called 
"tribofilm" [9-12]. Since the present "transfer films" are quite different from the initial material due to selective transfer phenomena, we will define these transfer layers as "tribofilms", like in the case of tribological products that are generated not only from the additives in oil lubricated conditions but also from thin solid films sliding in dry conditions. Indeed, the tribofilm consist mostly of $\mathrm{Cu}$ or $\mathrm{Ag}$ determined by energy dispersive X-ray spectroscopy (EDS), though the metal concentration in the Me-DLC is considerably lower than that in the tribofilms. These results indicate that the tribological properties of Me-DLC depend on the mechanical properties of metal-rich tribofilms, because the contact conditions in the frictional interface should vary depending on the chemical composition as well as the structure of the tribofilms. To understand the tribological properties of Me-DLC, it is necessary to clarify the formation process of metal-rich tribofilms on the sliding counterface.

In this study, the formation process of metal-rich tribofilm on the sliding counterface is presented using experimental results obtained from tribological experiments on $\mathrm{Cu}-\mathrm{DLC}$ and $\mathrm{Ag}-\mathrm{DLC}$.

\section{Experimental}

A Si $\left(\begin{array}{lll}1 & 0 & 0\end{array}\right)$ wafer with dimensions of $8 \times 10 \times 0.6$ $\mathrm{mm}^{3}$ was used as a substrate for Me-DLC coatings deposition. The Si $\left(\begin{array}{lll}1 & 0 & 0\end{array}\right)$ substrate was ultrasonically cleaned by organic alkaline solution, and then rinsed in distilled water several times. Water on the substrate was removed by clean nitrogen blow, and then the substrate was introduced in the coating chamber of Me-DLC. The Me-DLC coatings were deposited on the substrate using an hybrid deposition process composed of plasma enhanced chemical vapor deposition (PECVD) from acetylene/argon gas mixture and DC magnetron sputtering of metal target. The details of hybrid deposition process were reported elsewhere $[8,13]$. The mixture of $\mathrm{C}_{2} \mathrm{H}_{2}$ and Argon was introduced in the chamber, and the pressure was maintained at $1.33 \mathrm{~Pa}$ during the deposition process. The metal concentration in the coating was varied by changing the gas mixture ratio between $\mathrm{C}_{2} \mathrm{H}_{2}$ and $\mathrm{Ar}$, and was estimated by the composition ratio between metal and $\mathrm{C}$ in the film, as measured by EDS. The accuracy of quantitative analysis by EDS is lower than that by Wavelength Dispersive X-ray Spectroscopy (WDX), especially for light element such as C. In addition, EDS is unable to detect hydrogen, which is a component of Me-DLC in this study. The accuracy of the composition rate of Me-DLC might be lower than a few at.\%. Despite this relatively low accuracy, the metal concentration measured by EDS allows to compare the metal/carbon content ratio in the different films. As a result, several Me-DLC coatings with different concentrations were achieved by changing gas mixture ratio of acetylene and argon gases under a fixed DC input. In this paper, $\mathrm{Cu}-\mathrm{DLC}$ with $\mathrm{Cu}$

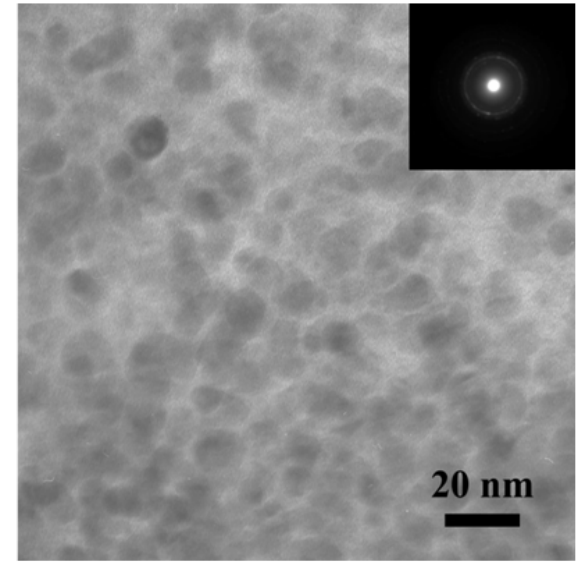

Fig. 1 TEM image of nano structure of Cu-DLC with a copper concentration of 50 at.\%

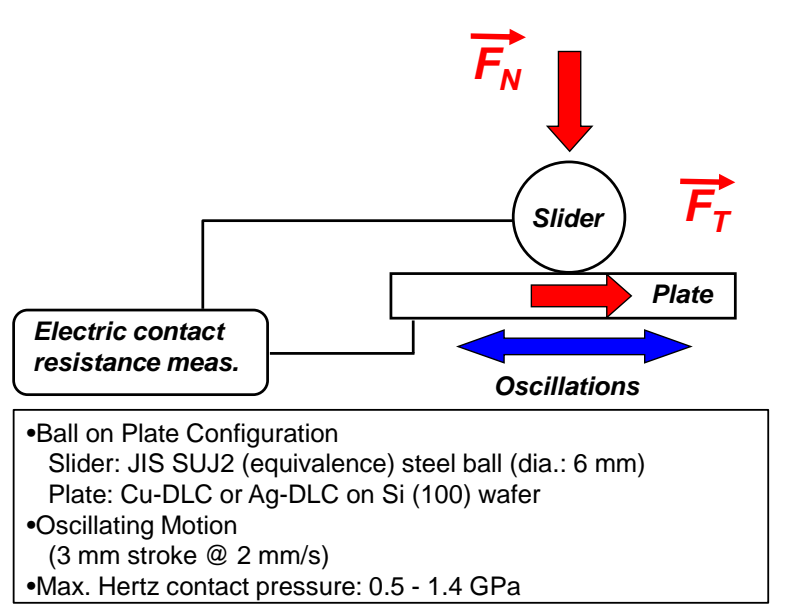

Fig. 2 Schematic diagram of tribotest configuration

concentration of 50 at. $\%$ and Ag-DLC with $\mathrm{Ag}$ concentration ranging from 63 to 84 at. $\%$ were investigated. By transmission electron microscopy (TEM), we observed that metal clusters are dispersed homogeneously in an amorphous DLC matrix. Figure 1 shows typical example of nanostructure of $\mathrm{Cu}$-DLC with a $\mathrm{Cu}$ concentration of 50 at.\%. The diameter of the $\mathrm{Cu}$ clusters is $5 \mathrm{~nm}$ in average. We found the tendency that the cluster diameter increases with increasing metal concentration in the film. The diameter of Ag clusters in Ag-DLC with Ag concentration of 75 at. $\%$ was approximately $20 \mathrm{~nm}$, which was reasonable result because the metal concentration in the Ag-DLC was much higher than that in Cu-DLC. The thickness of $\mathrm{Cu}$-DLC and Ag-DLC were about $1.2 \mu \mathrm{m}$, according to the previously measured deposition rate and the selected deposition time. The nanoindentation hardness of $\mathrm{Cu}-\mathrm{DLC}$ and Ag-DLC were respectively 4.3 and 1.8 GPa.

The linear reciprocating tribological experiments 


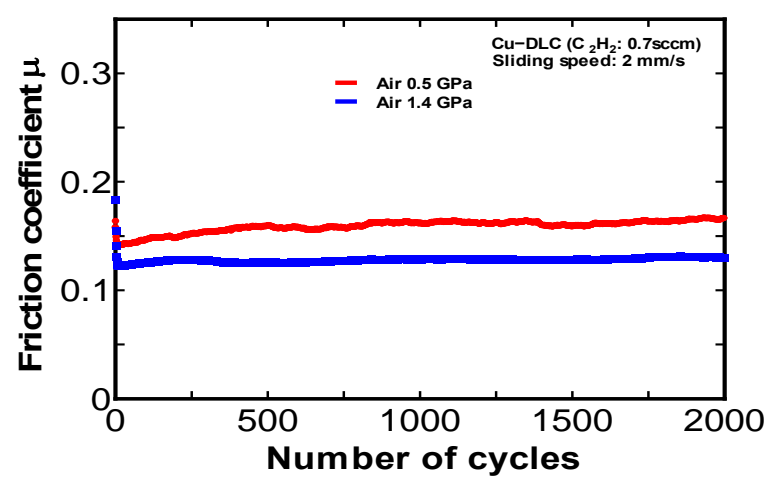

Fig. 3 Transition of friction coefficient of $\mathrm{Cu}-\mathrm{DLC}$

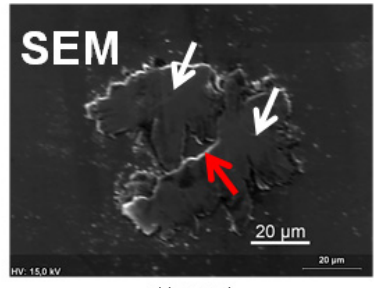

(a) SEM image

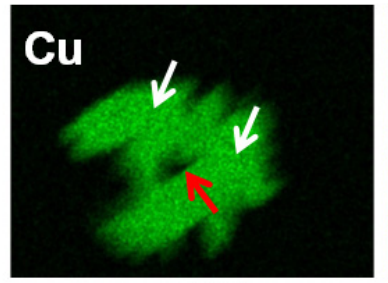

(c) $\mathrm{Cu} \mathrm{K \alpha}$

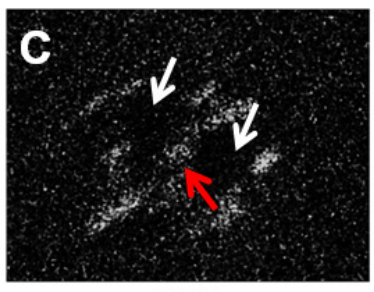

(b) $\mathrm{C} \mathrm{K \alpha}$

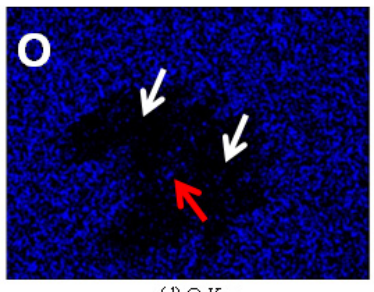

(d) $\mathrm{OK} \alpha$
Fig. 4 Characterization of $\mathrm{Cu}$-rich tribofilm by scanning microscopy SEM and EDS

were performed under a sliding speed of $2.0 \mathrm{~mm} / \mathrm{s}$ with a stroke of $3 \mathrm{~mm}$ for a wide range of contact pressure: 0.5 to $1.4 \mathrm{GPa}$. A ball-on-plate configuration was adopted for the experiment in atmospheric condition. Mirror polished bearing steel ball (JIS SUJ2 or equivalence) with a diameter of $6 \mathrm{~mm}$ was reciprocated on the Me-DLC surfaces. The configuration of the tribological experiments is schematically shown in Fig. 2. The electrical contact resistance (ECR) was also measured continuously during the friction tests.

\section{Results and discussion}

3.1. Cu-rich tribofilm in slider / Cu-DLC interface

Figure 3 shows the evolution of friction coefficient of $\mathrm{Cu}$-DLC under the maximum contact pressure of 0.5 and $1.4 \mathrm{GPa}$ in air, as a function of sliding cycles. After a short running-in period, the friction coefficient exhibited stable values at around 0.15 for both contact pressures. In both cases, a tribofilm with metallic luster was formed on the steel counterface, according to the observation by optical microscope (OM) after tribo-tests. This result indicated that $\mathrm{Cu}-\mathrm{DLC}$ provides similar
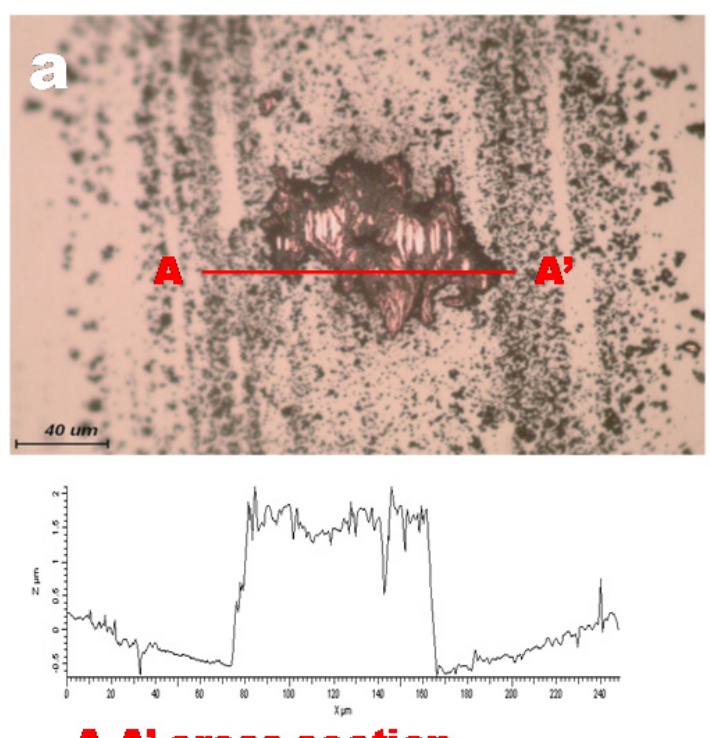

\section{A-A' cross section}

(a) $0.5 \mathrm{GPa}$
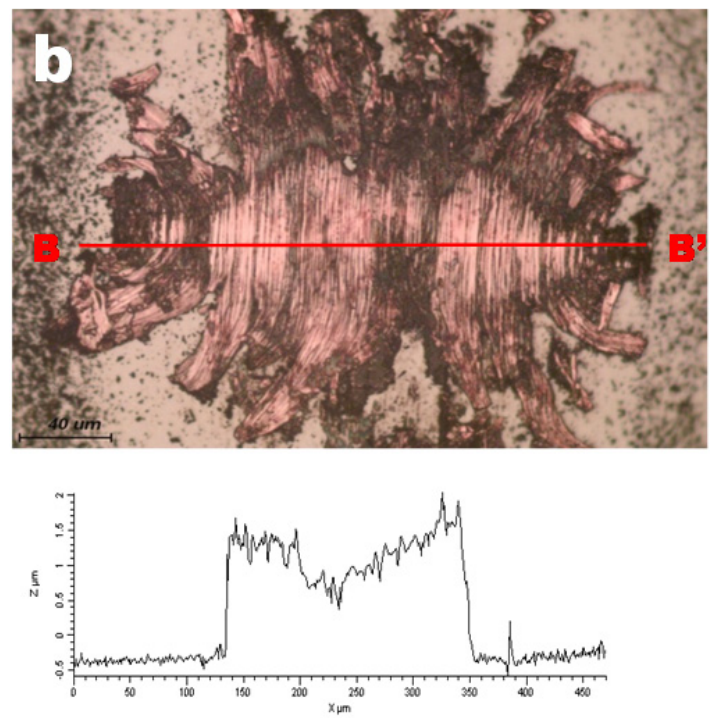

3-8' eross section

(b) $1.4 \mathrm{GPa}$

Fig. $5 \mathrm{Cu}$-rich tribofilms under the different loading conditions

friction coefficient under the different contact pressures, when the metallic-tribofilm forms on the counterface.

The relation between tribological performance of $\mathrm{Cu}-\mathrm{DLC}$ and its electrical performance has a trade-off relation [3]. The friction coefficient of $\mathrm{Cu}-\mathrm{DLC}$ increases as the increase of $\mathrm{Cu}$ concentration, whereas the ECR decreases as the $\mathrm{Cu}$ increase in the film. The ECR of the $\mathrm{Cu}$-DLC used in this report is the order of 1 $\mathrm{k} \Omega$.

Figure 4 shows the results of characterization on a typical tribofilm by scanning electron microscopy (SEM) and EDS, namely the tribofilm formed on 


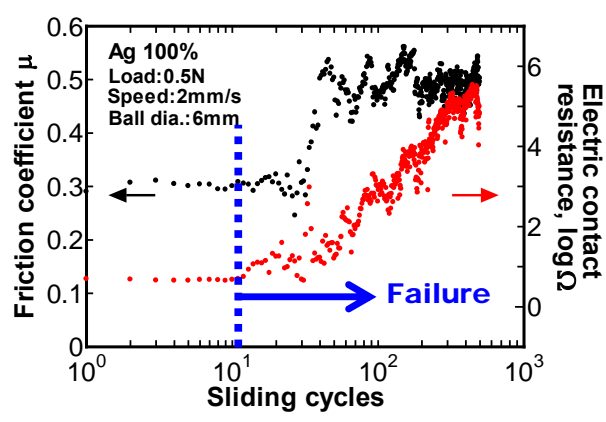

(a) Ag 100 at. $\%$

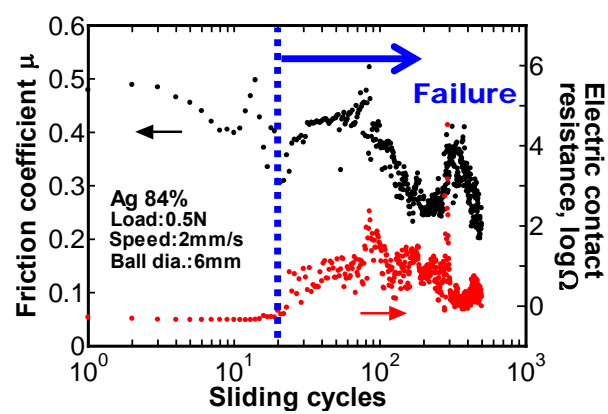

(b) Ag 84 at. $\%$

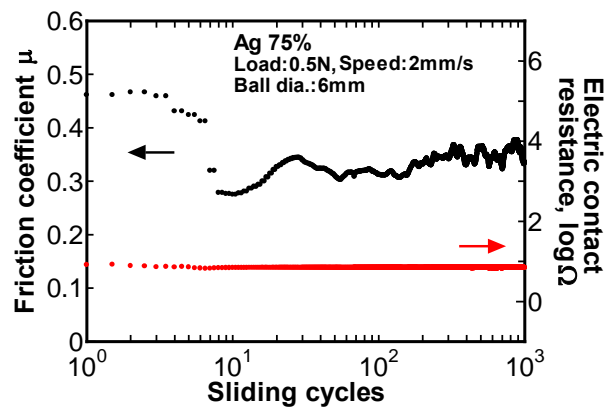

(c) Ag 75 at.\%

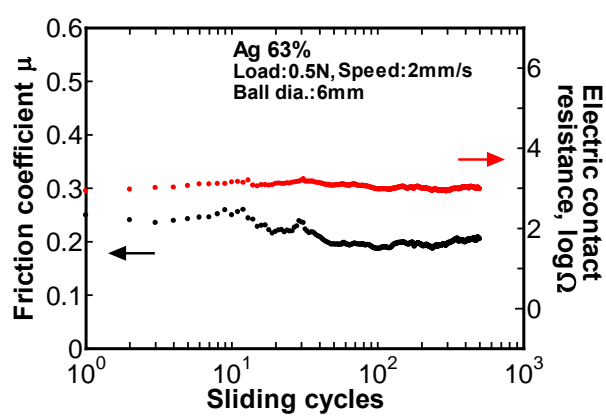

(d) Ag 63 at. $\%$

Fig. 6 Friction coefficients and ECR of Ag-DLCs under Hertz contact pressure of $0.5 \mathrm{GPa}$

bearing steel slider under the contact pressure of 0.5 GPa after 2000 cycles of sliding. The SEM image shows that the tribofilm consists of two segments shown by white arrows in Fig. 4. Indeed, $\mathrm{C}$ and $\mathrm{O}$ are both detected in the boundary between these segments (ex.: red-arrowed part) and edges, but the intensities of both $\mathrm{C} \mathrm{K} \alpha$ and $\mathrm{O} \mathrm{K} \alpha$ on the center part of the two segments (ex.: white-arrowed part) are much weaker than those on the surrounding steel surface, which was covered by both hydrocarbon contaminations and natural oxide layer (see Fig. 4(b) and (d)). In addition, Fig. 4(c) shows that these two segments emitted strong $\mathrm{Cu} \mathrm{K \alpha} \mathrm{X}$-ray during EDS analysis. These results indicate that the tribofilm consists of neither $\mathrm{Cu}$ oxide nor $\mathrm{Cu} / \mathrm{C}$ composite, but mostly $\mathrm{Cu}$ at a high concentration. It should be noted that the $\mathrm{Cu}$-rich tribofilm was hardly oxidized during tribo-test under atmospheric condition, although $\mathrm{Cu}$ is a strongly oxidizable metal. Moreover, the debris of carbon-based DLC matrix was removed from the tribofilm, and the purity of $\mathrm{Cu}$ was increasing. These results indicate that the frictional interface between $\mathrm{Cu}$-DLC and steel slider has acted as a characteristic reactor in which complex phenomena have been taking place.

Figure 5 shows $\mathrm{Cu}$-rich tribofilms formed on counterface of $\mathrm{Cu}$-DLC after 2000 sliding cycles under the different loading conditions. Corresponding initial Hertz contact pressures were $0.5 \mathrm{GPa}$ (Fig. 5(a)) and 1.4 GPa (Fig. 5(b)), respectively. The cross section profiles of the tribofilms are also provided in the figure by non-contact profilometry measurements. Many fragments of carbonaceous wear debris are surrounding the tribofilms. The morphology of the tribofilm varied from "mosaic structure" to "continuous metal plate" as the initial Hertz contact pressure increase. The morphological change of the tribofilm reflects the degree of plastic deformation of the tribofilm, and it can be noticed that large plastic flow occurs under the high contact pressure. The cross section profiles show that the thickness of tribofilm is estimated to be less than 2 $\mu \mathrm{m}$, and that the thickness decreases with increasing Hertz contact pressure. This result indicates that the shear rate of tribofilm at frictional interface becomes significantly high, which would be estimated more than $1000 \mathrm{~s}^{-1}$.

\subsection{Ag-rich tribofilm in slider / Ag-DLC interface}

The evolution of friction coefficient of Ag-DLC with various $\mathrm{Ag}$ concentrations under the initial Hertz contact pressure of $0.5 \mathrm{GPa}$ are shown in Fig. 6(a)-(d), together with ECR during sliding. The experimental data of pure Ag film by sputtering is also shown as a reference. The pure Ag film failed immediately after 10 cycles, but the durability increased with the decrease of $\mathrm{Ag}$ concentration, i.e. the increase of a DLC fraction. As far as the film durability was reaching 2000 cycles, a tribofilm with metallic luster formed on the steel counterface.

From the comparison between the two kinds of experimental data obtained from the Ag-DLCs with Ag concentration of less than 75 at.\% (see Fig. 6(c) and (d)), it is clear that the friction coefficient decreases with 


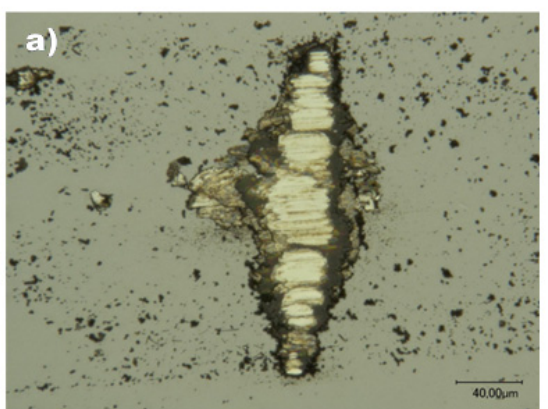

(a) $0.5 \mathrm{GPa}$

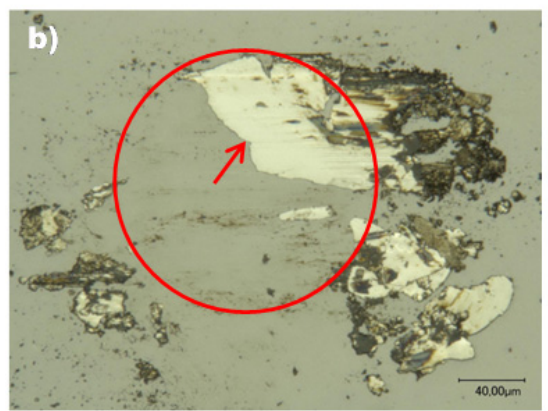

(b) $1.4 \mathrm{GPa}$

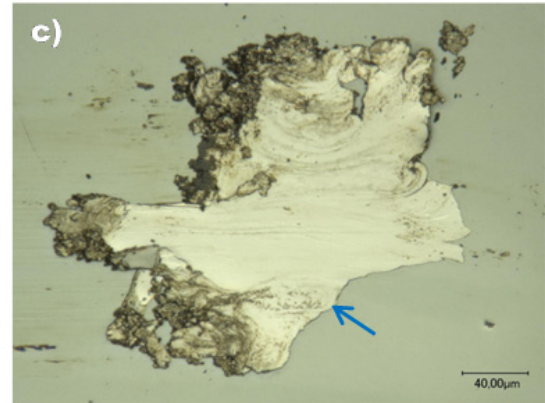

(c) detached part of tribofilm from (b) on Ag-DLC

Fig. 7 Optical images of two kinds of tribofilms

The shape of red-arrowed part in Fig. 9(b) corresponds to that of blue-arrowed part in Fig. 9(c)

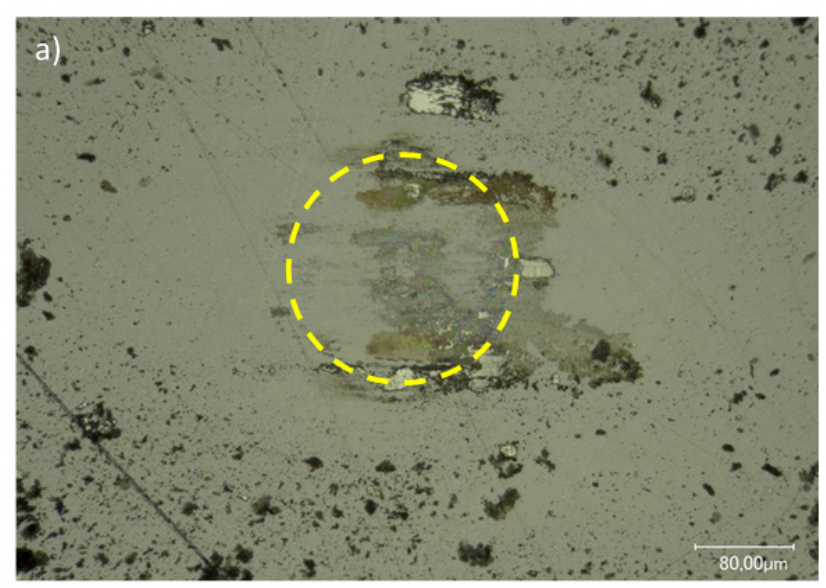

(a) wear scar of steel slider without Ag-rich tribofilm formation on the sliding surface

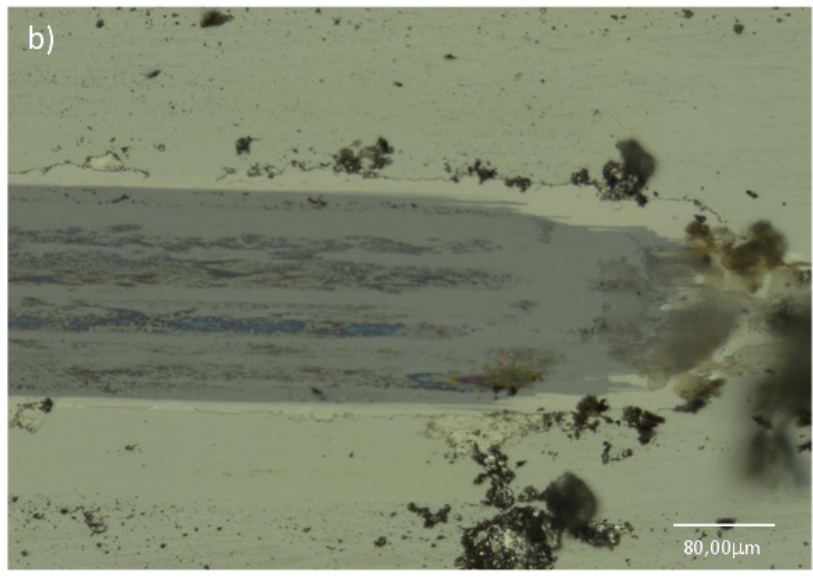

(b) wom track on Ag-DLC

Fig. 8 Wear scar of steel slider and the worn track on Ag-DLC, without Ag-rich tribofilm formation on the slider

increasing DLC fraction. This can be explained by the decrease of the Ag volume and the resulting increase of hardness of Ag-DLC. Thus, the increase of the DLC fraction in the film is effective to increase the durability of the Ag-DLC as well as the decrease of the friction coefficient. This is the same tendency as the characteristics of $\mathrm{Cu}$-DLC, which was discussed previously $[3,4]$.

On the other hand, a superfluous increase of the DLC fraction causes the increase of the ECR of Ag-DLC. The ECR reached less than $10 \Omega$ when the Ag concentration was 75 at.\%, but it increased up to the order of $1 \mathrm{k} \Omega$ when the $\mathrm{Ag}$ concentration decreased to 63 at.\% (see Fig. 6(c) and (d)). This means that the increasing $\mathrm{Ag}$ concentration in the film is necessary to improve the electrical resistivity of the Ag-DLC, but it is the opposite way to improving the film durability and reducing friction coefficient. Therefore, the optimization $\mathrm{Ag}$ concentration in the Ag-DLC is an important parameter to obtain excellent tribological performance and preferable electrical properties, which is exactly the same tendency as the trade-off correlation of $\mathrm{Cu}$-DLC.

Figure 7 shows the OM images of two kinds of tribofilms formed under the contact pressure of 0.5 and $1.4 \mathrm{GPa}$. Since the adhesive strength of the tribofilm originating from $\mathrm{Ag}$-DLC on bearing steel was weak compared to the one of the $\mathrm{Cu}$-rich tribofilm, a part of it was detached from steel surface and remained on the worn track of Ag-DLC, as shown in Fig. 7(c)). When the tribofilm was completely detached from the slider surface, the Ag-DLC was worn out rapidly. Figure 8 shows the OM images of the wear scar on the steel slider and the worn track on Ag-DLC, without tribofilm formation on the slider. In this case, Ag-DLC was worn away at 400 sliding cycles. This means that the tribofilm formation on the counterface is necessary to obtain a good tribological performance. The approximate contact area in Fig. 7(b) is shown by a red-circle on the picture. 

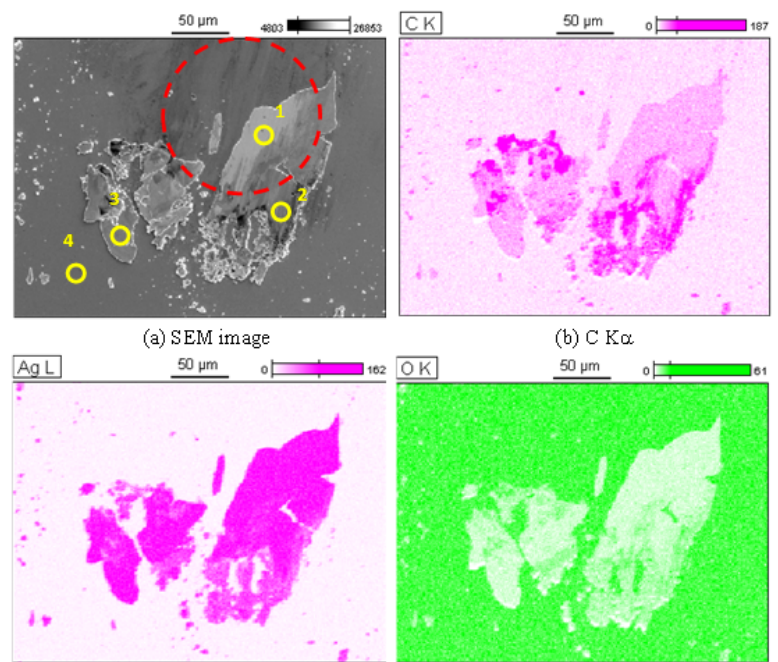

(c) Ag L $\alpha$

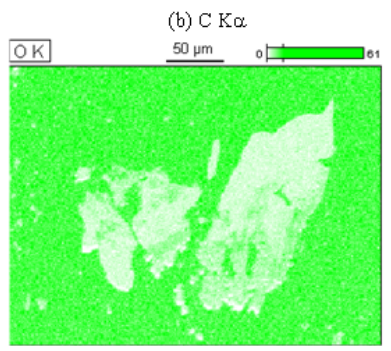

(d) $\mathrm{OK} \alpha$

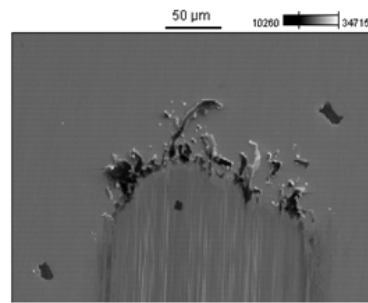

(a) SEM image

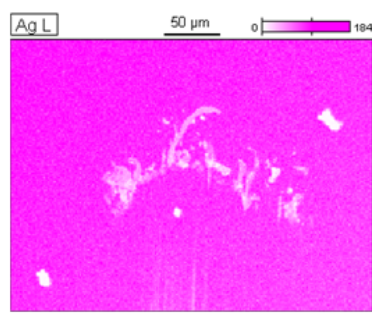

(c) Ag L $\alpha$

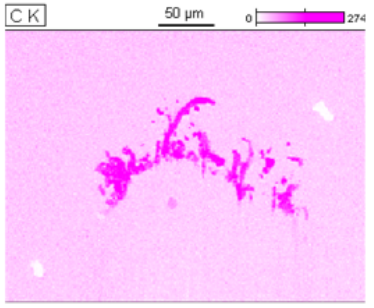

(b) $\mathrm{C} \mathrm{K \alpha}$

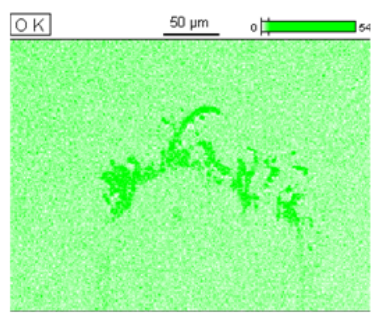

(d) $\mathrm{O} \alpha$

Fig. 9 Characterization of Ag-rich tribofilm by SEM and EDS

Fig. 10 SEM image and chemical maps of the typical example of worn track end on Ag-DLC

Table 1 Chemical composition of Ag-rich tribofilm

\begin{tabular}{|c|c|c|c|c|c|c|}
\hline \multirow{2}{*}{$\begin{array}{c}\text { Location } \\
\text { No. }\end{array}$} & \multicolumn{7}{|c|}{ Composition, at.\% } \\
\cline { 2 - 7 } & $\mathrm{C}$ & $\mathrm{O}$ & $\mathrm{Si}$ & $\mathrm{Cr}$ & $\mathrm{Fe}$ & $\mathrm{Ag}$ \\
\hline 1 & 7.6 & 0.0 & 0.3 & 0.0 & 0.0 & 92.1 \\
\hline 2 & 15.5 & 16.1 & 0.0 & 0.0 & 0.0 & 68.4 \\
\hline 3 & 9.5 & 1.9 & 0.0 & 0.0 & 0.0 & 88.6 \\
\hline 4 & 3.5 & 0.0 & 0.7 & 1.4 & 94.4 & 0.0 \\
\hline
\end{tabular}

The frictional surfaces on both tribofilm show metallic luster, but the outside of the contact area shows dark or black color. The morphology of the tribofilm originating from $\mathrm{Ag}$-DLC is similar to $\mathrm{Cu}$-rich tribofilm with respect to the Hertz contact pressure, but the shape of the tribofilm is different from the one of $\mathrm{Cu}$-rich tribofilm under low contact pressure.

Figure 9 shows the results of characterization of the tribofilm on the slider surface formed under an Hertz contact pressure of $1.4 \mathrm{GPa}$ (see Fig. 7(b)), by SEM and EDS. The contact area is visualized by a red-circle in the SEM image. Colors in the chemical maps of $\mathrm{C} \mathrm{K \alpha}$, $\mathrm{Ag} \mathrm{L} \alpha$ and $\mathrm{O} \mathrm{K} \alpha$ indicate a high amount of these elements, while white color indicates a small amount. The tribofilm and its fragments contain high amounts of Ag as shown in Fig. 9(c). Thus, the tribofilm originating from Ag-DLC is Ag-rich tribofilm. On the other hand, the tribofilm hardly contain $\mathrm{C}$ and $\mathrm{O}$ in its main part. Indeed $\mathrm{C}$ and $\mathrm{O}$ exist in the border of the tribofilm and its fragments, but their intensities are very weak in the central region of the tribofilm, in the center of the contact circle where the Hertz contact pressure is highest (see Fig. 9(b) and (d)). The results of quantitative analysis on the tribofilm are shown in Table 1. The location number corresponds to the numbers of the yellow-circles in Fig. 9(a). The location number 4 is a virgin surface of the steel slider, as a reference. Oxygen was detected in the border of tribofilm and its fragment, but was not detected in the tribofilm at the center of the contact circle (location No. 1) as well as on the slider surface. The bearing steel surface was covered by an oxide layer, but the concentration of $\mathrm{O}$ was under the quantitative limits of the EDS analysis in this condition. Figure $9(\mathrm{~d})$ shows that the concentration of $\mathrm{O}$ on the main part of the tribofilm (No. 1) is obviously lower than the one on the oxidized steel surface. This means that the Ag-rich tribofilm was not oxidized. On the other hand, $\mathrm{C}$ was detected from all analyzed point, but the concentration at the border of the tribofilm and in the fragments (No. 2 and No. 3) is much higher than 


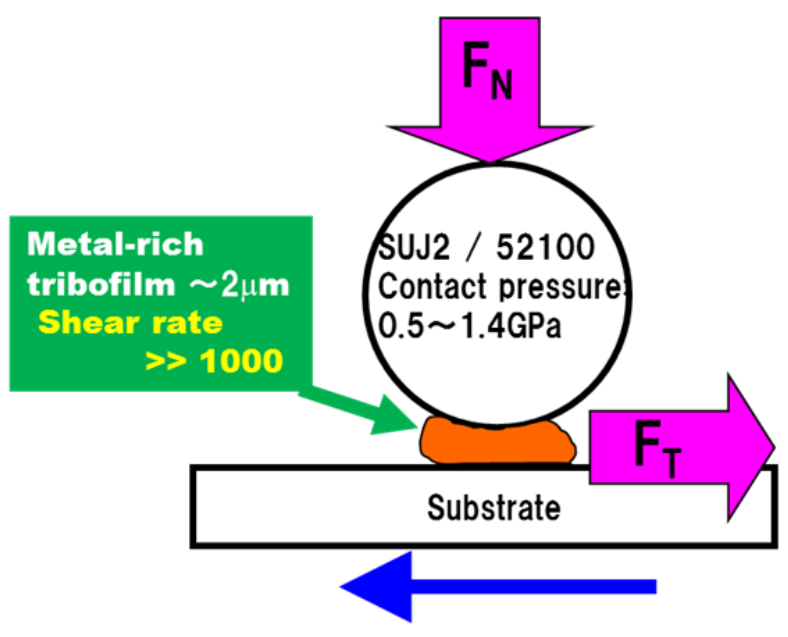

Fig. 11 Schematic image of cross section of the contact point between Me-DLC and slider

in the main part of tribofilm (No. 1) or on the steel surface (No. 4). Carbon is one of the components of hydrocarbon contaminations on solid surface, and is always detected on surface analysis excepting after special-surface-cleaning such as annealing in ultrahigh vacuum. Since the fluorescence quantum yield of $\mathrm{C}$ is low because of low atomic number, the quantitative accuracy of $\mathrm{C}$ is considerably lower than that of transition metals by EDS analysis. In addition, the difference of background of X-rays on silver and on steel should affect the contrast in the chemical map of Fig. 9(b) since the backscattered electron yield of Ag is higher than that of steel, because the backscattered electrons have sufficient energy to excite $\mathrm{C}$ atoms in the contaminant layer as well as direct incident electrons. Figure 10 shows SEM image and chemical maps of a typical example of a worn track end on Ag-DLC. Wear debris are scattered around the end of the worn track on Ag-DLC, shown in Fig. 10(a). The wear debris consists of $\mathrm{C}$ and $\mathrm{O}$ without $\mathrm{Ag}$. This means that the wear debris forms either $\mathrm{C} / \mathrm{O}$ composite or $\mathrm{C}$ oxide. These results indicate that the main part of Ag-rich tribofilm consists mostly of Ag without neither oxide nor carbide, and that the fragment and border of the tribofilm outside of contact area contained $\mathrm{C}$ and $\mathrm{O}$ as either $\mathrm{C} / \mathrm{O}$ composite or $\mathrm{C}$ oxide.

\subsection{Formation process of metal-rich tribofilm}

The origin of the tribofilm is the metal clusters, i.e. $\mathrm{Ag}$ clusters or $\mathrm{Cu}$ clusters in the Me-DLC. Figure 11 illustrates the cross section of the contact point between Me-DLC and slider via metal-rich tribofilm during sliding. The clusters in wear debris agglomerate selectively on the counterface, and then the agglomerated clusters formed an "almost pure $\mathrm{Cu}$ or $\mathrm{Ag}$ tribofilm", even in an oxidizable environment. In the case of Ag-rich tribofilm, it is reasonable that $\mathrm{Ag}$ clusters agglomerate without oxidation under oxidizable atmosphere, since Ag oxides are an unstable phase and are easy to deoxidize by heating in air. The Gibbs free energy of $\mathrm{Ag}$ oxides is higher than that of pure $\mathrm{Ag}$ under temperatures higher than room temperature [14]. On the other hand, it is very surprising that the $\mathrm{Cu}$, which is known as a highly oxidizable metal, formed an almost non-oxidized $\mathrm{Cu}$-rich tribofilm under oxidizing environment. Actually, $\mathrm{Cu}$ clusters didn't undergo the oxidation, and gathered as "tribofilm" without $\mathrm{C}$ debris.

The sintering of oxidizable metal powder can be possible, which was reported by several researchers [15, 16]. Indeed titanium is well known as a highly oxidizable metal, but solidification of $\mathrm{Ti}$ powder has been reported by Nakayama et al. under the combined conditions of compression with rotating shear motion [15]. Miki et al. reported that the solidification of aluminum powder under combined conditions of high compression and shear motion [16]. These results indicate that the oxidizable metal powder can be sintered under combined conditions of compression and shear motions.

In the case of the formation process of $\mathrm{Cu}$-rich tribofilm, since the $\mathrm{Cu}$ clusters underwent high compressive pressure up to $1.4 \mathrm{GPa}$ as well as high shear rate over $1000 \mathrm{~s}^{-1}$, the $\mathrm{Cu}$ clusters on the slider surface were considered to be sintered together in the frictional interface which generates high pressure and high shear rate. On the other hand, since $\mathrm{C}$ may form neither $\mathrm{Cu}$ carbide nor $\mathrm{Ag}$ carbide, and the density of the $\mathrm{C}$ debris is considerably smaller than that of $\mathrm{Cu}$ and $\mathrm{Ag}$, the $\mathrm{C}$ debris would be removed effectively out of the tribofilm by repeated plastic deformations in the frictional interface. Moreover, some $\mathrm{C}$ clusters from the debris are able to react with $\mathrm{O}$ atoms, and this reaction could yield $\mathrm{C}$ oxides such as friction polymers that are also smaller density than that of both $\mathrm{Ag}$ and $\mathrm{Cu}$. The result of EDS analysis, showing that the concentration of Ag at the center of the contact area was higher than at the outskirts as shown in Figs. 9 and 10, is supporting the above discussion. In addition, the nanoindentation hardness of $\mathrm{Cu}$-rich tribofilm is around $2 \mathrm{GPa}$ with some effects of piling up around indents, under the different Hertz contact pressures of both 0.5 and 1.4 $\mathrm{GPa}$. From the above discussed sintering process and the result of nanoindentation hardness of $\mathrm{Cu}$-rich tribofilm, the contact pressure in the real contact area between $\mathrm{Cu}$-rich tribofilm / Cu-DLC would become almost constant, i.e., real contact area becomes proportional to the normal load, and the shear strength of the real contact area might be almost constant under the different Hertz contact pressures. Therefore, the friction coefficients showed almost same values under the two different Hertz contact pressures.

In conclusion, the adhered metal clusters on the counterface, undergoing iterative plastic deformation under high pressure and high shear rate. As a result, the metal in the tribofilm is gradually refined and sintered in the frictional interface. 


\section{Conclusion}

Metal-rich tribofilms are formed on the counterface of both $\mathrm{Cu}-\mathrm{DLC}$ and Ag-DLC during sliding, and are almost pure $\mathrm{Cu}$ and $\mathrm{Ag}$, according to EDS measurements. The morphology of metal-rich tribofilms varied from mosaic structure to continuous-plate structure as the contact pressure increase. The formation process of metal-rich tribofilms is concluded that the adhered $\mathrm{Cu}$ and $\mathrm{Ag}$ clusters on the counterfaces are possibly sintered and refined during iterative plastic deformation in the frictional interface which generates high pressure and high shear rate.

\section{Acknowledgements}

This work was partly supported by Grant-in-Aid for Scientific Research (C) (23560174) and (C) (26420093) of Japan Society for the Promotion of Science (JSPS). Part of the work was carried out under the Collaborative Research Project (J13083, J14038) of the Institute of Fluid Science, Tohoku University. This work was partly supported by the JSPS Core-to-Core Program, A. Advanced Research Networks, "International research core on smart layered materials and structures for energy saving". This project is performed within the framework of ELyT Lab. (France-Japan joint laboratory), involving the following institutions: CNRS French National Center for Scientific Research, ECL Ecole Centrale de Lyon, INSA de Lyon (FRANCE), Tohoku University (JAPAN).

\section{References}

[1] Donnet, C. and Erdemir, A. (Eds.), "Tribology of Diamond-Like Carbon Films - Fundamentals and Application," 2008, Springer.

[2] Goto, M. and Honda, F., "Film-Thickness Effect of Ag Lubricant Layer in the Nano-Region," Wear, 256, 2004, 1062-1071.

[3] Fontaine, J., Belin, M., Takagi, T., Miki. H., Adachi, K., Takeno, T., Goto, M. and Ito, K., "Tribological Behavior and Electrical Contact Resistance of Metal-Containing DLC Coatings for Electrically Conductive Tribo-Elements," Proc. AFI/TFI CRF-36, Sendai, November 2010, 106-107.

[4] Goto, M., Fontaine, J., Bec, S., Ito, K., Takeno, T., Ruet, M., Miki, H., Belin, M. and Le Mogne, T., "The Role of Tribofilm Growth on the Frictional Behavior of Copper-Diamond-Like Carbon Nanocomposite Coating," Proc. 38th Leeds-Lyon Symposium on Tribology 2011, Lyon, September 2011.

[5] Goto, M., Ruty, N., De Chillaz, V., Fontaine, J., Takeno, T., Miki, H. and Belin, M., "Silver-Diamond-Like Carbon Nanocomposite Coatings: Tribological Behavior and Electrical
Capabilities," Proc. ITC Hiroshima 2011, Hiroshima, October 2011, E1-01.

[6] Goto, M., Fontaine, J., Bec, S., Belin, M., Le Mogne, T., Ito, K., Takeno, T. and Miki, H., "Tribological Properties of Me-DLC Containing $\mathrm{Ag}$ and $\mathrm{Cu}$," Proc. Eighth International Conference on Flow Dynamics, Sendai, November 2011, 644-645.

[7] Goto, M., Fontaine, J., Takeno, T. and Miki, H., "Tribological Properties of Ag-DLC Under Various Loading Conditions," Proc. Ninth International Conference on Flow Dynamics, Sendai, September 2012, 770-771.

[8] Takeno, T., Saito, H., Goto, M., Fontaine, J., Miki, H., Belin, M., Takagi, T. and Adachi, K., "Deposition, Structure and Tribological Behavior of Silver - Carbon Nanocomposite Coatings," Diamond and Rerated Materials, 39, 2013, 20-26.

[9] Biswas, S. K., "Some Mechanisms of Tribofilm Formation in Metal/Metal and Ceramic/Metal Sliding Interactions," Wear, 245, 2000, 178-189.

[10] Martin, J. M, Grossiord, C, Le Mogne, T, Bec, S. and Tonck, A., "The Two-Layer Structure of Zndtp Tribofilms: Part I: AES, XPS and XANES Analyses," Tribology International, 34, 2001, 523-530.

[11] Fontaine, J., "Towards the Use of Diamond-Like Carbon Solid Lubricant Coatings in Vacuum and Space Environments," Proceedings of the Institution of Mechanical Engineers, Part J: Journal of Engineering Tribology, 222, 2008, 1015-1029

[12] Osterle, W., Dmitriev, A. I., Gradt, T., Hausler, I., Hammouri, B., Morales Guzman, P. I., Wetzel, B, Yigit, D. and Zhang, G., "Exploring the Beneficial Role of Tribofilms Formed from an Epoxy-Based Hybrid Nanocomposite," Tribology International, $88,2015,126-134$.

[13] Takeno, T., Hoshi, Y., Miki, H. and Takagi, T., "Activation Energy in Metal-Containing DLC Films with Various Metals of Various Concentrations," Diamond and Related Materials, 17, 2008, 1669-1673.

[14] Michaelides, A., Bocquet, M. -L., Sautet, P., Alavi, A. and King, D. A., "Structures and Thermodynamic Phase Transitions for Oxygen and Silver Oxide Phases on Ag $\left\{\begin{array}{llll}1 & 1 & 1\end{array}\right\}, "$ Chemical Physics Letters, 367, 2003, 344-350.

[15] Nakayama, N., Kato, S., Takeishi, H. and Miki, H., "Consolidation of Ti Powder by a Compression Rotation Shearing System at Room Temperature -Effect of Pivot Rotation Speed on Consolidation," Advanced Material Research, 409, 2012, 3-8.

[16] Miki, H., Nakayama, N. and Takeishi, H., "Dynamic Molding of Powder Particles at Room Temperature," Materials Science Forum, 2012, 706-709, 1955-1960. 